conventional infrared (IR) imaging of a bonded wafer, it is difficult to determine whether a lack of visible voids on the interface corresponds to strong bonding. Using dynamic surface energy measurement, researchers Xuanxiong Zhang of Shanghai Research Center for Wireless Communication in China and Jean-Pierre Raskin of Université Catholique de Louvain in Belgium have studied bonding strength and bonding uniformity of bonded wafer pairs before and after post-bonding annealing. Low-temperature $\mathrm{O}_{2}$ plasma exposure was used to bond Si samples (3 in. diameter, $<100>$-oriented, $p$-type, $15-25 \Omega \mathrm{cm}$ ) that had been pre-cleaned using a standard procedure and then exposed to $\mathrm{O}_{2}$ plasma for $5 \mathrm{~s}$. The researchers discovered that, overall, the bonding strength increased significantly after $10 \mathrm{~h}$ of annealing at $120^{\circ} \mathrm{C}$.

As the researchers reported in the August issue of Electrochemical and Solid-State Letters (p. G268; DOI: 10.1149/ 1.2012288 ), some areas seemingly free of voids in IR images have much lower surface energy values than the mean value, indicating that these areas may be de-bonding or have voids. After $20 \mathrm{~h}$ of annealing, the overall bonding strength is improved further, but the non-uniformity observed earlier still exists. With longer annealing times of up to $120 \mathrm{~h}$, the bonding strength rose further by a small amount, but the uniformity of the bonding strength is remarkably enhanced. In particular, in almost all tested samples, the bonding strength at the rim of a bonded pair is higher than in the center of the bonded pair.

The researchers believe that the increase of bonding strength and the enhancement of bonding strength uniformity over the annealing time may be due to out-diffusion of bonding by-products (i.e., hydrogen, oxygen, and water vapor). Therefore, they conclude that extended post-bonding annealing is necessary to obtain uniform bonding strength on the whole wafer level and improve the percentage of successful bonding yield in practical applications.

SHIMING WU

\section{Spa Water Used for the Fabrication of Visible-Light-Emitting Porous Silicon}

Visible light emission from porous silicon has potential application in silicon-based optoelectronic devices. Most porous Si (p-Si) layers are prepared by anodic etching on $p$-type Si wafers or photoetching $p$-type Si wafers in an HF solution. Both methods have disadvantages involving either complicated fabrication steps or special handling of toxic chemicals. A group of researchers in Japan have now used potable spa water as the solution in photoetching to produce visible-light-emitting p-Si.

As reported in the August issue of Electrochemical and Solid-State Letters (p. G251; DOI: 10.1149/1.2001791), S. Adachi and K. Tomioka from the Department of Electronic Engineering of Gunma University in Japan have fabricated visible-light-emitting porous Si by photoetching Si in spa water. The spa water they used is a colorless alkaline solution from one of the Japanese hot springs, the Tenkeisen Spa. The researchers photoetched an $n$-type $\mathrm{Si}(111)$ wafer in the Tenkeisen Spa water (TeSW) under $5 \mathrm{~mW}$ He$\mathrm{Ne}$ laser $(632.8 \mathrm{~nm})$ illumination for $3 \mathrm{~h}$ with a laser spot size of $\sim 1 \mathrm{~mm}$. They proposed that the photochemical etching in Si took place in a two-step reaction: first electron-hole pairs were generated in the Si by optical excitation. Hole aggregation near the $\mathrm{Si}$-solution interface produced $\mathrm{Si}^{2+}$ ions; the ionic $\mathrm{Si}^{2+}$ then reacted with $\mathrm{OH}^{-}$to form $\mathrm{Si}_{x}(\mathrm{OH})_{y}$ complexes, which are soluble in the TeSW. The researchers also anodized a $p$-type $\mathrm{Si}(100)$ wafer in an ethanolic HF electrolyte for comparison. Both p-Si samples showed a red photoluminescence peak at $1.95 \mathrm{eV}$.

The structural and optical properties of the TeSW-prepared p-Si sample are nearly the same as those prepared in HF solution, although neither $\mathrm{H}^{+}$nor $\mathrm{F}^{-}$is present in the spa water. The thick-

\section{Precision Heating} to1200 $\mathrm{C}$ in 24 seconds, for under $10 \mathrm{~K}$ ?

\section{NOW THAT'S HOT!}

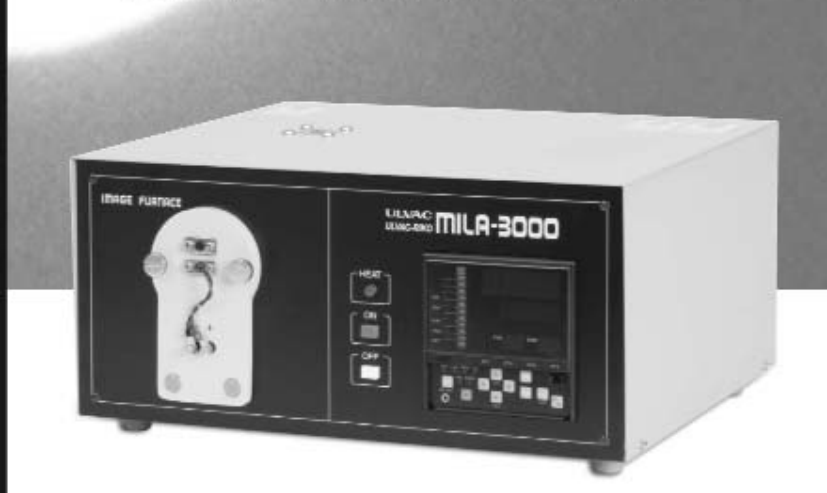

Anneal your small samples faster and with better control using the programmable MILA-3000 tabletop furnace. Ulvac's Mini-Lamp Annealing System can rapidly heat and cool samples with it's infrared gold image furnace, providing precision high temperature control, clean heating and versatile atmosphere selection.

\section{MILA-3000 Features:}

- High controlled heating rates of $50^{\circ} \mathrm{C} / \mathrm{s}$

- Sample size $20 \times 20 \times 20 \mathrm{~mm}$

- Air, vacuum and inert/reactive gas atmosphere

- Temperature uniformity of $+/-2^{\circ} \mathrm{C}$

- Low power consumption (1 kW)

For all your annealing and thermal processes, turn on the heat with the MILA-3000!

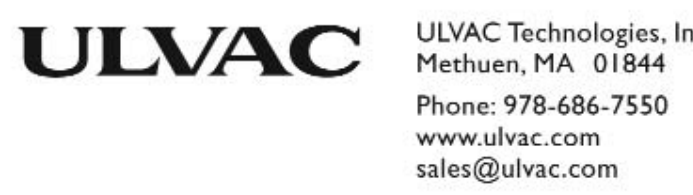


ness of the TeSW-prepared p-Si is very thin and the rms roughness is only $1.6 \mathrm{~nm}$.

The researchers also performed a controlled experiment of $\mathrm{p}$-Si formation using $\mathrm{NaOH}$ etchant at various $\mathrm{pH}$ levels, but they observed no efficient PL emission from any of the $\mathrm{NaOH}$-prepared samples. The researchers pointed out that the reproducibility appeared to be a problem for the photoetched p-Si synthesis technique. They point out that others have suggested that the preexisting state of the $\mathrm{Si}$ surface plays a decisive role in determining $\mathrm{p}-\mathrm{Si}$ formation.

TAO XU

\section{High Frictional Anisotropy Found on Quasicrystal Surface}

A collaboration of scientists from Lawrence Berkeley National Laboratory (Berkeley Lab) and the Ames Laboratory at Iowa State University have determined that friction along the surface of a quasicrystal in the direction of a periodic geometric configuration is about eight times greater than in the direction where the geometric configuration is aperiodic (i.e., without regularity). In an article published in the August 26 issue of Science (p. 1354), the research collaboration led by Miquel Salmeron, a physicist with Berkeley Lab's Materials Sciences Division, and Patricia Thiel, a chemist in Ames Lab, reports that geometric periodicity was confirmed by rows of atoms that formed a Fibonacci sequence, a numerical pattern often observed in quasicrystals. The research combined the use of scanning tunneling microscopy (STM) and atomic force microscopy (AFM) to observe the surface structures and measure the frictional forces.

"That we can get such a large difference in frictional force just by scratching the surface of a material in a different direction was a major surprise," said Salmeron. "Our results reveal a strong connection between interface atomic structure and the mechanisms by which frictional energy is dissipated."

At the atomic level, when two surfaces come in contact, the chemical bonds and clouds of electrons in their respective atoms create frictional force and cause energy to be dissipated. It has long been known that friction is greater between surfaces of identical crystallographic orientation than between surfaces of differ- ing orientation. Some recent studies have reported frictional differences, or anisotropy, less than a factor of 3 for incommensurate crystal surfaces when there were periodicity differences.

To measure the frictional effects due to periodicity alone and not to other factors such as chemical differences, the researchers worked with decagonal quasicrystals of an aluminum-nickel-cobalt (Al$\mathrm{Ni}-\mathrm{Co}$ ) alloy. Stacked planes of Al-Ni-Co crystals exhibit both tenfold and twofold rotational symmetry. By cutting a single Al-Ni-Co quasicrystal parallel to its tenfold axis, the researchers were able to produce a two-dimensional surface with one periodic axis and one aperiodic axis, separated by $90^{\circ}$. In order to avoid surface damage and also wear of the tip used in the combined AFM-STM instrument, a TiN tip passivated with a molecular layer of hexadecane thiol was used.

"Strong friction anisotropy was observed when the AFM tip slid along the two directions: high friction along the periodic direction, and low friction along the aperiodic direction," said co-researcher J.Y. Park of Berkeley Lab. "We believe the source of this friction has both an electronic and a phononic contribution." Phonons are quantized vibrations in a crystal lattice. The frictional anisotropy vanished when the samples were oxidized by exposure to air.

The researchers said that new theoretical models are needed to determine whether the electronic or phononic contributions dominate the observed frictional anisotropy.

\section{La-Doped 0.3PZN-0.7PZT Ceramics with Pure Perovskite Phase Synthesized}

It is well known that the single-crystal relaxor ferroelectrics, $\mathrm{Pb}\left(\mathrm{Zn}_{1 / 3} \mathrm{Nb}_{2 / 3}\right) \mathrm{O}_{3}$ $\mathrm{PbTiO}_{3}(\mathrm{PZN}-\mathrm{PT})$ and $\mathrm{Pb}\left(\mathrm{Mg}_{1 / 3} \mathrm{Nb}_{2 / 3}\right) \mathrm{O}_{3}$ $\mathrm{PbTiO}_{3}$ (PMN-PT), possess ultrahigh piezoelectric properties. However, in comparison with single crystals, ceramics have advantages including low production cost, homogeneous compositions, tunable properties for applications such as medical ultrasonic probes and microfine actuators. Although pure perovskite PMN-PT ceramics have been synthesized near their morphotropic phase boundary (MPB), until now success has eluded such efforts for PZN-PT. In the August issue of the Journal of the American Ceramic Society (p. 2310; DOI: 10.111/j.15512916.2005.00391.x), G. Deng and colleagues from the Shanghai Institute of Ceramics, China, have reported their success in synthesizing pure perovskite ceramics of PZN-PZT, as an alternative to PZN-PT, near their MPB. The researchers have observed much higher piezoelectric and electromechanical coupling coefficients for their newly synthesized material than those reported earlier.

Because their goal was to find good piezoelectric values, the researchers explored $0.3 \mathrm{~Pb}\left(\mathrm{Zn}_{1 / 3} \mathrm{Nb}_{2 / 3}\right) \mathrm{O}_{3}-0.7 \mathrm{~Pb}_{0.96} \mathrm{La}_{0.04}$ $\left(\mathrm{Zr}_{x} \mathrm{Ti}_{1-x}\right)_{0.99} \mathrm{O}_{3}$ [La-doped 0.3PZN0.7PZT], with $x$ ranging from 50 to 53 stepwise by 0.5 (i.e., values around the MPB). The samples were prepared by the two-step hot-pressing process. X-ray diffractometry $(X R D)$ revealed that the microstructures of these complex compounds experience a gradual transition process from tetragonal to rhombohedral phase with an increase in the $\mathrm{Zr} / \mathrm{Ti}$ ratio. The MPB is defined as the coexistence of tetragonal and rhombohedral phases. Therefore, the research team deduced that the MPB in their samples can be delimited in the range of $\mathrm{Zr} / \mathrm{Ti}=$ 51/49-52/48. In addition, XRD revealed that samples in the entire composition range had pure perovskite structure. The piezoelectric coefficient $\left(d_{33}\right)$ was measured using a quasi-static piezoelectric $d_{33}$ meter. The maximum of $d_{33}$ was found to be $845 \mathrm{pC} / \mathrm{N}$ for $\mathrm{Zr} / \mathrm{Ti}=51 / 49$, which the researchers indicate is much higher than the previously reported value of 670 $\mathrm{pC} / \mathrm{N}$. Electromechanical coupling was measured using an impedance analyzer, and its maximum value was observed to be 0.7 for the same composition $\mathrm{Zr} / \mathrm{Ti}=$ $51 / 49$ and larger than the previously reported value of 0.65 . Dielectric coefficient and dielectric loss were measured using the impedance analyzer. This series of piezoelectric ceramics have high $T_{\max }$ $\left(206-213^{\circ} \mathrm{C}\right)$ and large dielectric constants (3321-4088 for poled and 2048-2796 for depoled samples). The researchers concluded that these high values of piezoelectric properties make 0.3PZN-0.7PLZT an interesting material for potential applications in transducers, actuators, and probes.

VIVEK RANJAN

\section{MRS Materials Connections} w w w. mrs.org/connections / Your source for Materials Research-Related Information! 